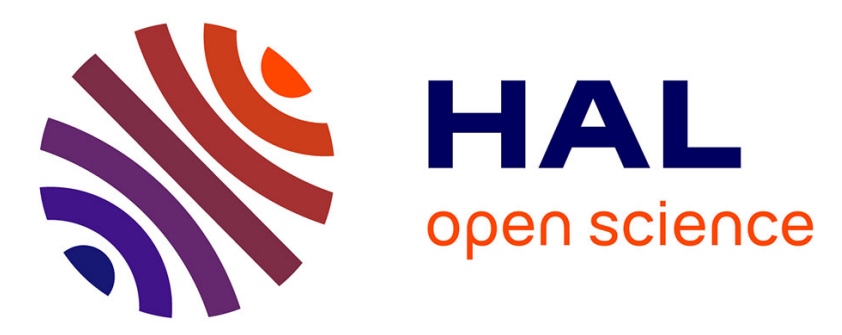

\title{
Influence des facteurs métallurgiques sur quelques propriétés magnétiques du nickel polycristallin
}

\author{
Bernard Dubois
}

\section{To cite this version:}

Bernard Dubois. Influence des facteurs métallurgiques sur quelques propriétés magnétiques du nickel polycristallin. Revue de Physique Appliquée, 1970, 5 (4), pp.669-681. 10.1051/rphysap:0197000504066900 . jpa-00243443

HAL Id: jpa-00243443

https://hal.science/jpa-00243443

Submitted on 1 Jan 1970

HAL is a multi-disciplinary open access archive for the deposit and dissemination of scientific research documents, whether they are published or not. The documents may come from teaching and research institutions in France or abroad, or from public or private research centers.
L'archive ouverte pluridisciplinaire HAL, est destinée au dépôt et à la diffusion de documents scientifiques de niveau recherche, publiés ou non, émanant des établissements d'enseignement et de recherche français ou étrangers, des laboratoires publics ou privés. 


\title{
INFLUENCE DES FACTEURS MÉTALLURGIQUES SUR QUELQUES PROPRIÉTÉS MAGNETTIQUES DU NICKEL POLYCRISTALLIN
}

\author{
Par Bernard DUBOIS (*)
}

(Reçu le 8 avril 1970)

\begin{abstract}
Résumé. - Les propriétés magnétiques du nickel dépendent d'une part de la pureté du métal et d'autre part des traitements mécaniques et thermiques.

Pour un nickel pur bien recuit, les propriétés magnétiques sont en fait déterminées par la teneur en atomes résiduels de soufre. Cependant la mise en forme, la taille de l'échantillon, la température et le temps de recuit, la grosseur du grain... sont des facteurs importants dont nous avons précisé le rôle. Outre quelques essais de traînage magnétique, nous avons souligné l'incidence du magnétisme du nickel sur sa résistivité électrique à basse température et sur sa capacité d'amortissement interne.
\end{abstract}

Abstract. - Magnetic properties of nickel depend on the one hand of purity of metal and on the other hand of mechanical and thermal treatments. In a well annealed pure nickel, residual atoms of sulfur determine magnetic properties. Nevertheless cold work, geometrical dimensions, temperature and annealing time, grain size... are important factors that we have explicited. In addition to some after-effect measurements, we have underlined the influence of magnetic properties of nickel on its low temperature resistivity and on its internal friction.

Si les connaissances fondamentales en métallurgie progressent grâce à l'utilisation de monocristaux, la pratique courante met en œuvre soit le métal brut de déformation, soit le métal simplement recuit. Dans ce dernier cas, pour un métal de pureté donnée, les propriétés vont dépendre en particulier de la taille $\mathrm{du}$ grain. Nous avons voulu savoir, à propos du nickel, métal isotrope, dans quelle mesure la grosseur de grain était susceptible d'influencer les propriétés magnétiques du métal. En effet, nous pouvions envisager de faire varier cette grandeur en jouant soit sur la pureté du métal, soit sur les traitements mécaniques et thermiques qui déterminent la texture de l'échantillon. De plus nous nous sommes proposés de rechercher les influences respectives, sur les propriétés magnétiques, de la taille de grain, des impuretés résiduelles et des dimensions de l'échantillon. Pour mener à bien une telle étude nous avons envisagé de mettre en œuvre l'observation micrographique des domaines de Weiss et surtout les mesures magnétiques classiques sur fils. A la suite de telles études il devait être possible de préciser dans quelles conditions les propriétés magnétiques sont un critère de pureté du nickel. Enfin nous nous sommes demandés si les propriétés magnétiques exercent une influence sur la résistivité électrique à basse température et sur la capacité d'amortissement interne du nickel.

(*) Laboratoire de Métallurgie E. N. S. C. P. 11, rue Pierre et Marie-Curie, Paris 5 e. La majeure partie du travail expérimental a été effectuée au Laboratoire de Vitry du Professeur Chaudron.
1. Propriétés étudiées et méthodes expérimentales [1]. - Nous rappelons ici les propriétés magnétiques qui ont retenu notre attention en renvoyant à des publications antérieures pour les précisions techniques.

1.1 Mise en ÉVIDENCE Des DOMAINES FERROMAGNÉTIQUES. - Le nickel possède 8 directions de facile aimantation du type $\left\langle\begin{array}{lll}11 & 1\end{array}\right\rangle$ et des parois à $180^{\circ}$, $109^{\circ} 28^{\prime}$ et $70^{\circ} 32^{\prime}$ séparent les domaines qui apparaissent sur les surfaces ayant des orientations privilégiées. Sur le métal massif, la méthode d'observation la plus courante est celle des figures de Bitter. Une suspension colloïdale de magnétite [2] est déposée sur l'échantillon préalablement désaimanté dans un champ alternatif d'amplitude décroissante. Au bout d'un temps assez long - du fait de la faible anisotropie du nickel - la structure magnétique apparaît. Pour réussir la mise en évidence des domaines, il faut disposer d'échantillons bien recuits, polis électolytiquement et exempts de contraintes mécaniques. Le dépôt de magnétite se fait à l'aide d'une pipette et un circuit magnétique [3] permet l'observation de la modification des domaines sous l'effet d'un champ. Cependant le déplacement des parois est tributaire de l'inertie des particules de magnétite. De plus, la floculation ou le séchage peuvent intervenir de manière catastrophique. Nous envisageons actuellement la mise en œuvre de l'effet Kerr qui permet de suivre le comportement des parois en fonction de la température jusqu'au point de Curie [4]. 
1.2 Courbe DE PREMIÈre aIMANTATION ET CYCle D'HYSTÉRÉSIS. - La courbe de première aimantation et le cycle d'hystérésis du nickel ont été soit enregistrés à l'aide d'un magnétomètre, soit tracés point par point à l'aide d'un fluxmètre. Ces méthodes sont classiques [3] mais un certain nombre de corrections sont nécessaires dans le cas des fils [1]. Les études sur couronnes ont été réalisées au moyen d'un perméamètre à bobines démontables. A la suite des mesures effectuées en courant continu nous pourrons accéder, par exemple, aux grandeurs suivantes :

- perméabilité initiale $\mu_{0}$,

- pente de la droite de Rayleigh $v$,

- limite supérieure du domaine de validité de la loi de Rayleigh $H_{\mathrm{R}}$ (domaine dans lequel la perméabilité varie linéairement avec le champ appliqué),

- perméabilité maximale $\mu_{\max }$,

- champ correspondant à la perméabilité maximale $H \mu_{\text {max }}$,

- induction à saturation $B_{\mathrm{s}}$ (si l'excitation magnétique est suffisante),

- induction à la rémanence $B_{\mathrm{r}}$,

- champ coercitif $H_{\mathrm{c}}$.

1.3 MagnÉtostriction. - Des mesures de magnétostriction ont été effectuées sur des nuances de nickel que nous avons préparées. L'appareillage et le détail des manipulations ont été décrits par ailleurs [5]

1.4 EsSAIS DE TRAîNAGE MAGNÉTIQUE. - Des essais de traînage magnétique ont été effectués dans un dispositif du type transformateur ouvert $[6,7]$ déjà utilisé pour l'étude du carbone en solution dans le fer.
2. Influence de la pureté du nickel. - Le nickel se prépare industriellement soit par électrolyse, soit par décomposition du nickel tétracarbonyle (procédé Mond). Ce dernier procédé assure une meilleure séparation du cobalt mais le métal est enrichi en carbone (tableau I). Il est intéressant de comparer les propriétés de ces métaux à celles de nickels purifiés par fusion de zone $[1,8]$. Cependant le traitement de fusion de zone n'est pas efficace pour éliminer le cobalt et le fer, aussi il doit être effectué sur un dépôt électrolytique provenant d'une solution de chlorure de nickel traitée sur résine échangeuse d'ions [9].

2.1 ANAlyse DES NUANCES ÉTUDIÉES. - Nous indiquons dans le tableau I, pour les principales nuances de nickel étudiées, d'une part les teneurs en impuretés les plus notables et d'autre part les valeurs des propriétés les plus remarquables. Les dosages sont effectués par spectrographie de masse et par radioactivation [10] mais le carbone est dosé par chromatographie en phase gazeuse [11] et le soufre par voie chimique [12]. La température de recristallisation est déterminée à l'aide des rayons $X$ sur des échantillons laminés de $95 \%$. Remarquons que cette température est identique pour le nickel électrolytique $B$ et pour le nickel $\mathrm{ZF}_{22} \mathrm{E}$, bien que ce dernier métal présente un rapport de résistivités $\rho$ plus élevé, du fait de la teneur importante en cobalt. Pour les métaux $\mathrm{ZF}_{22} \mathrm{C}$ et $\mathrm{ZF}_{8} \mathrm{~L}$, nous obtenons une diminution parallèle de $\rho$ et de la température de recristallisation. Dans le cas des propriétés magnétiques, que nous allons examiner maintenant, les valeurs sont généralement données pour du nickel recuit à $1200^{\circ} \mathrm{C}$ sous argon pendant $12 \mathrm{~h}$, l'écrouissage préalable étant supérieur à $90 \%$.

TABLEAU I

Teneurs en impuretés (exprimées en $10^{-6}$ ) et propriétés principales des nuances de nickel étudiées.

\begin{tabular}{|c|c|c|c|c|c|c|c|c|c|c|c|c|c|}
\hline & Co & $\mathrm{Fe}$ & As & $\mathrm{Cu}$ & $\mathbf{S}$ & O & C & $\begin{array}{l}\text { Tempéra- } \\
\text { ture de re- } \\
\text { cristallisa- } \\
\text { tion } \theta_{R}{ }^{\circ} \mathrm{C}\end{array}$ & $\left\{\begin{array}{l}\rho_{H}= \\
10^{4} \cdot \frac{\rho 20,3 \mathrm{~K}}{\rho 294 \mathrm{~K}} \times 10^{4}\end{array}\right.$ & $\left|\begin{array}{l}\rho_{H e}= \\
10^{4} \frac{\rho}{\rho 294^{\circ} \mathrm{K}}\end{array}\right|$ & $\mu_{\max }$ & $\begin{array}{c}H_{c} \\
(\mathrm{Oe})\end{array}$ & $\begin{array}{l}\text { Gros- } \\
\text { seur de } \\
\text { grain } \\
(\mu \mathrm{m})\end{array}$ \\
\hline $\begin{array}{c}\text { Ni électro A } \\
\downarrow \\
\mathrm{NiZF}_{22} \mathrm{E}\end{array}$ & $\left|\begin{array}{l}5300 \\
4500 \text { à } \\
7300\end{array}\right|$ & $\begin{array}{l}1000 \\
1100\end{array}$ & $\begin{array}{l}350 \\
3,6\end{array}$ & $\begin{array}{l}140 \\
\text { à } 220 \\
165 \text { à } \\
185\end{array}$ & $\begin{array}{l}<10 \\
1,5 \mathrm{à} \\
4,5\end{array}$ & $\begin{array}{l}80 \\
0 \text { à } \\
2\end{array}$ & $\begin{array}{l}27 \pm 5 \\
7 \pm 2\end{array} \mid$ & $\begin{array}{l}600 \\
370\end{array}$ & $\begin{array}{l}980 \\
585(*) \\
365\end{array}$ & $\begin{array}{l}- \\
-\end{array}$ & $\begin{array}{c}- \\
5700\end{array}$ & $\begin{array}{l}1,9 \\
0,15\end{array}$ & $\begin{array}{r}50 \\
600\end{array}$ \\
\hline Ni électro B & 250 & $\begin{array}{l}84 \text { à } \\
130\end{array}$ & 3 à 5 & 65 à 90 & $\begin{array}{l}10 \mathrm{à} \\
30\end{array}$ & & $11 \pm 2$ & 370 & 130 & - & 3100 & 0,30 & 300 \\
\hline $\begin{array}{c}\overline{\text { Ni ex-carbonyle }} \\
\downarrow \\
\mathrm{NiZF}_{22} \mathrm{C}\end{array}$ & $\begin{array}{l}6,9 \\
5,3 \text { à } \\
7,8\end{array}$ & $\begin{array}{l}93 \\
93 \text { à } \\
102\end{array}$ & $\left|\begin{array}{l}0,04 \\
0,02 a ̀ \\
0,07\end{array}\right|$ & $\begin{array}{l}37 \text { à } 60 \\
12 \pm 2\end{array}$ & $\begin{array}{l}14 \text { à } \\
40 \\
2,5 \text { à } \\
7\end{array}$ & $<1$ & $\begin{array}{l}52 \pm 7 \\
9 \pm 2\end{array}$ & $\begin{array}{l}350 \\
315\end{array}$ & $\begin{array}{l}135 \text { à } 118(*) \\
70 \\
48 \text { à } 55\left(^{*}\right) \\
50\end{array}$ & $\begin{array}{l}93(*) \\
33(*)\end{array}$ & $\begin{array}{l}2100 \\
6200\end{array}$ & $\begin{array}{l}0,6 \\
0,15\end{array}$ & $\begin{array}{l}180 \\
500\end{array}$ \\
\hline $\begin{array}{c}\text { Ni électro H.P. } \\
\downarrow \\
\mathrm{NiZF}_{8} \mathrm{~L}\end{array}$ & $\begin{array}{l}<0,09 \\
<0,1\end{array}$ & $\begin{array}{r}0,1 \\
<0,07\end{array}$ & 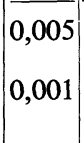 & $\begin{array}{l}1,47 \\
1,87\end{array}$ & & & $6 \pm 1$ & 260 & $\begin{array}{l}29 \text { à } 33(*) \\
22 \text { à } 31 \\
23 \text { à } 25(*) \\
\quad 20\end{array}$ & $\begin{array}{c}19\left(^{*}\right) \\
8,8 \text { à } 17 \\
10(*)\end{array}$ & $\begin{array}{l}5000 \\
6200\end{array}$ & $\begin{array}{l}0,17 \\
0,16\end{array}$ & $\begin{array}{l}300 \\
800\end{array}$ \\
\hline
\end{tabular}

(*) Mesures effectuées après recuit à $450^{\circ} \mathrm{C}$ sous vide. 
2.2 INFLUENCE DU DEGRÉ DE PURETÉ DU NICKEL SUR SES PROPRIÉTÉS MAGNÉTIQUES. - 2.2.1 Aspect des domaines ferromagnétiques. - Les micrographes 1 et 2 mettent en relief l'effet de la pureté globale du nickel sur l'aspect des murs de Bloch qui délimitent les domaines de Weiss. Brisées et perturbées par les

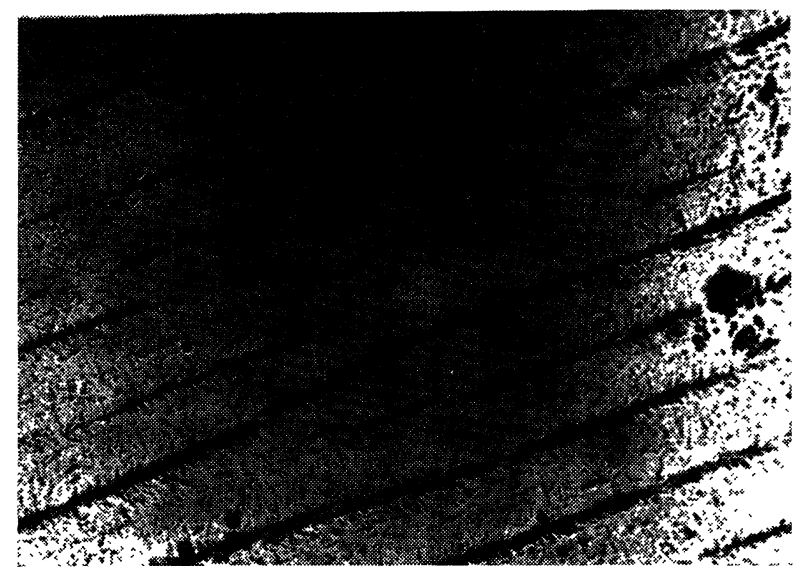

FIG. 1. - Nickel électrolytique B.G $\times 250$.

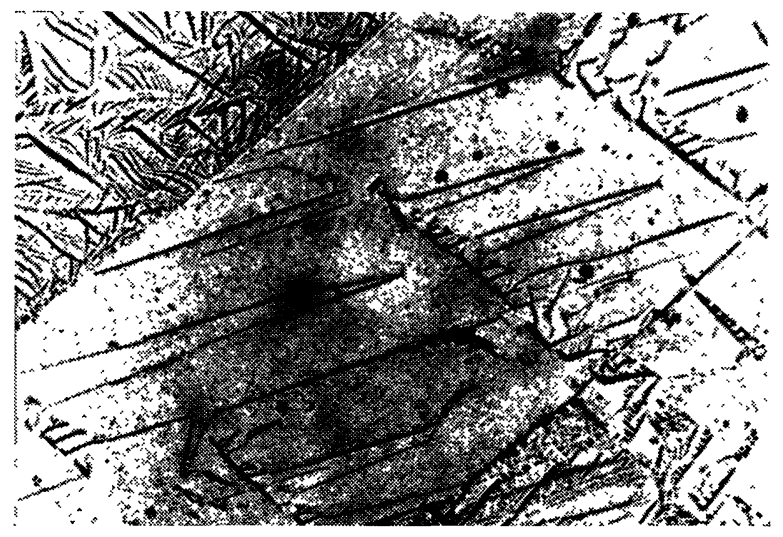

Fig. 2. - Nickel de zone fondue $\mathrm{ZF}_{22} \mathrm{C} . \mathrm{G} \times 100$.

inclusions et les défauts dans le métal industriel, les parois se présentent fines et régulières dans le métal purifié par fusion de zone (même si les teneurs en cobalt et en fer sont élevées). Sur le nickel de haute pureté, nous avons pu observer les interactions entre parois et joints de grains (ou joints de macle), les piques de Néel, etc... Au cours de ces observations nous avons noté pour toutes les nuances de nickel, une faible proportion de parois à $109^{\circ}$, ce qui laisse penser à une faible proportion de plans (110) à la surface de nos plaquettes [1]. Pour révéler les orientations des grains de façon rapide, nous avons mis au point un réactif donnant des figures de corrosion [13]. Sur des plans proches de (110), la largeur des domaines, varie de $250 \mu \mathrm{m}$ à $60 \mu \mathrm{m}$ quand on passe du nickel $Z Z F_{22} \mathrm{C}$ au métal électrolytique $\mathrm{B}$. Toutefois, il aurait été intéressant de faire une étude systématique analogue à celle de Bloor et Martin [14] pour effectuer des comparaisons à taille grain identique : ici la taille de grain était $0,8 \mathrm{~mm}$ dans le cas du métal $\mathrm{ZF}_{22} \mathrm{C}$ et $0,35 \mathrm{~mm}$ dans le cas du métal $\mathrm{B}$. Un aspect également intéressant de l'étude micrographique est la modification de la structure en domaines sous l'effet d'un champ magnétique : le déplacement apparaît faible et morcelé dans le cas du métal B (Fig. 3) tandis

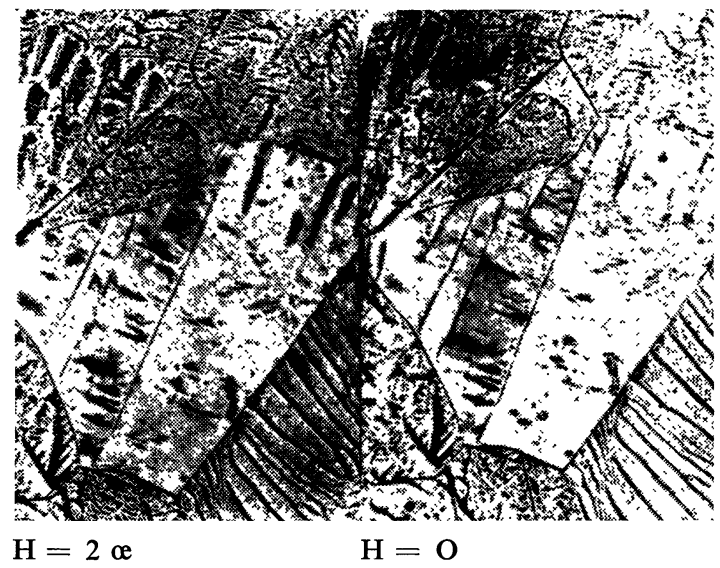

Fig. 3. - Mouvements des parois dans le nickel électrolytique B.G $\times 200$. Remarquez les faibles perturbations créées dans la région $\mathbf{A}$.

que des sauts de parois importants ou des changements brusques de structure se produisent dans le cas du métal de zone fondue (Fig. 4). Dans ce dernier métal, nous avons pu mesurer une amplitude de saut de $25 \mu \mathrm{m}$ pour une excitation de 1 œrsted.

a)

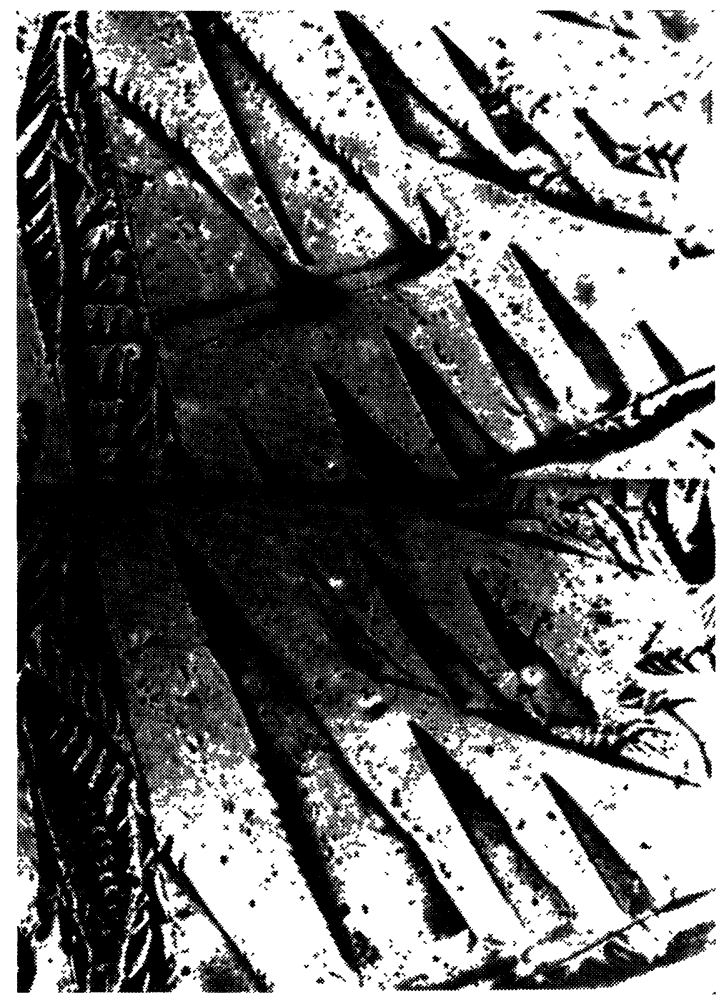

FIG. 4. - Nickel de zone fondue $\mathrm{ZF}_{22} \mathrm{E}$. $(\mathrm{G} \times 200)$.

a. - Etat désaimanté.

b. - Modification de la structure magnétique par application d'un champ élevé. 
2.2.2 Caractéristiques magnétiques usuelles du nickel. - Nous avons comparé les courbes de premières aimantations relatives au nickel d'origine carbonyle et au même métal traité par 22 passages de fusion de zone. Les mesures sont faites sur fils de diamètre $1,2 \mathrm{~mm}$, recuits $12 \mathrm{~h}$ à $1200^{\circ} \mathrm{C}$ sous argon. Il apparaît tout de suite une perméabilité beaucoup plus élevée dans le cas du métal de fusion de zone (Fig. 5) :

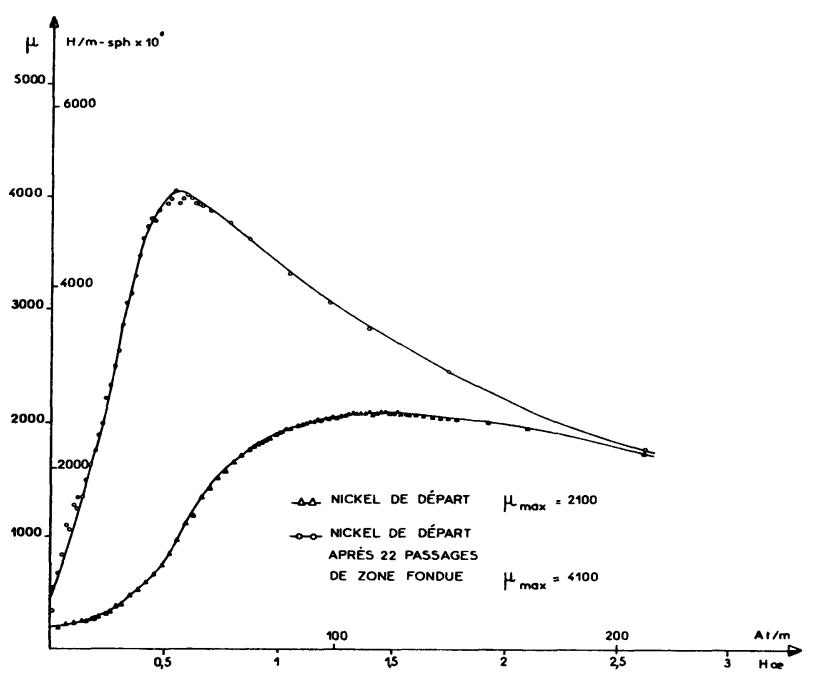

(a)

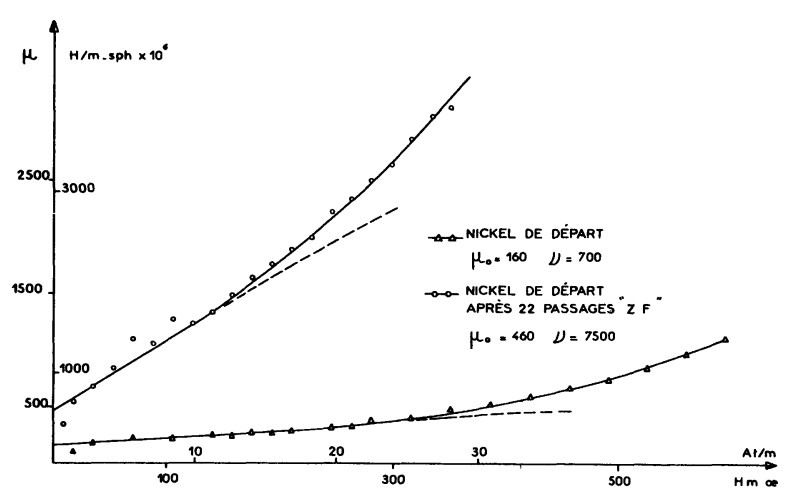

(b)

FIG. 5. - Comparaison des caractéristiques magnétiques du nickel d'origine carbonyle avant et après fusion de zone.

d'une part la perméabilité maximale $\mu_{\max }$ passe de 2100 à 4100 et d'autre part, la perméabilité initiale $\mu_{0}$ passe de 160 à 460 . La diminution de la teneur en impuretés, en particulier carbone et soufre, est mise en évidence par les dosages mais le rapport $\rho_{H}=\rho 20,3 \mathrm{~K} / \rho 294 \mathrm{~K}$ ne diminue que de $60 \times 10^{-4}$ à $48 \times 10^{-4}$. Il y a donc lieu de prendre en compte également la taille de grains qui varie de 180 à $300 \mu \mathrm{m}$ lorsque l'on passe d'une nuance à l'autre. Aussi pour mettre en évidence l'influence de la pureté du nickel sur ses propriétés magnétiques, il faut jouer sur l'écrouissage et sur le traitement thermique pour avoir des tailles de grain aussi comparables que possible. Dans le tableau II, nous donnons pour des tailles de grain différentes, les résultats obtenus par les caractéristiques du domaine de Rayleigh et pour le champ coercitif $H_{\mathrm{c}}$, la perméabilité maximale $\mu_{\max }$ et le champ correspondant $H \mu_{\text {max }}$. Examinons tout d'abord les résultats issus du domaine de Rayleigh. L'état désaimanté est important et nous avons toujours effectué la désaimantation au moyen d'un courant alternatif d'amplitude lentement décroissante. Mais d'après Jackson et coll. [15] on ne peut être sûr d'obtenir toujours la même configuration initiale des domaines. Toutefois, bien que toutes les mesures aient été effectuées dans les mêmes conditions nous n'avons pas fait d'étude systématique de l'influence du temps écoulé entre la fin de la désaimantation et le début de la mesure. Les valeurs obtenues indiquent que, pour une même taille du grain, on a sensiblement des valeurs plus élevées de $\mu_{0}$ et $v$ dans le cas des métaux de zone fondue. D'ailleurs, pour une nuance de nickel donnée il n'y a pas de variation régulière de ces caractéristiques avec la taille de grain. Les causes de dispersion des valeurs de $\mu_{0}$ et $v$ n'ont pas été trouvées même au moyen d'essais sur couronnes [1]. Remarquons que dans le cas de $v$, en particulier, la dispersion dépasse largement les erreurs commises lors de la détermination de cette pente. Nous pouvons rapprocher cette dispersion des valeurs rapportées par Kahan [16] pour la susceptibilité initiale $2<\chi_{0}<11$. Dans sa théorie des lois de l'aimantation de Lord Rayleigh, L. Néel [17] suppose que, dans le domaine étudié, la variation de l'aimantation produite par rotation réversible de l'aimantation à l'intérieur des domaines doit être faible devant celle qui provient des déplacements des parois. Il est alors possible de trouver une relation entre $\mu_{0}, v$ et $H_{\mathrm{c}}$. Ainsi sur un même barreau de fer de zone fondue, Dabosi [3] a montré au moyen des mesures au pont de Maxwell que $v / \mu_{0}$ était constant. Dans nos expériences, nous trouvons des valeurs dispersées de $\nu H_{\mathrm{c}} / \mu_{0}$ sauf peut être pour le nickel de fusion de zone $Z_{2} F_{22} C$. Il se pourrait donc que le nickel se prête mal à l'étude des déplacements de parois dans les faibles champs, par suite d'une intervention rapide des phénomènes de rotation.

Les résultats précédents sont d'autant plus surprenants que les valeurs de $\mu_{\max }, H \mu_{\max }$ et $H_{\mathrm{c}}$ déterminées sur les mêmes fils (partie gauche du tableau) sont beaucoup plus cohérentes. A grosseur de grain égale la perméabilité $\mu_{\max }$ est la plus élevée dans les métaux traités par fusion de zone : or globalement, d'après le tableau $\mathrm{I}$ le métal $\mathrm{ZF}_{22} \mathrm{E}$ est moins pur que le métal électolytique $B$ (ce qui est confirmé par la résistivité). Donc nous entrevoyons une influence spécifique de certaines impuretés sur les propriétés magnétiques : l'examen des analyses nous incite à penser aux métalloïdes (soufre, carbone...). En effet, les métaux $\mathrm{ZF}_{22} \mathrm{E}$ et $\mathrm{ZF}_{22} \mathrm{C}$ possèdent des teneurs du même ordre en ces éléments. Corrélativement le champ coercitif $H_{\mathrm{c}}$ et le champ correspondant à la perméabilité maximale $H \mu_{\max }$ sont les plus faibles. Nous 


\section{TABLEaU II}

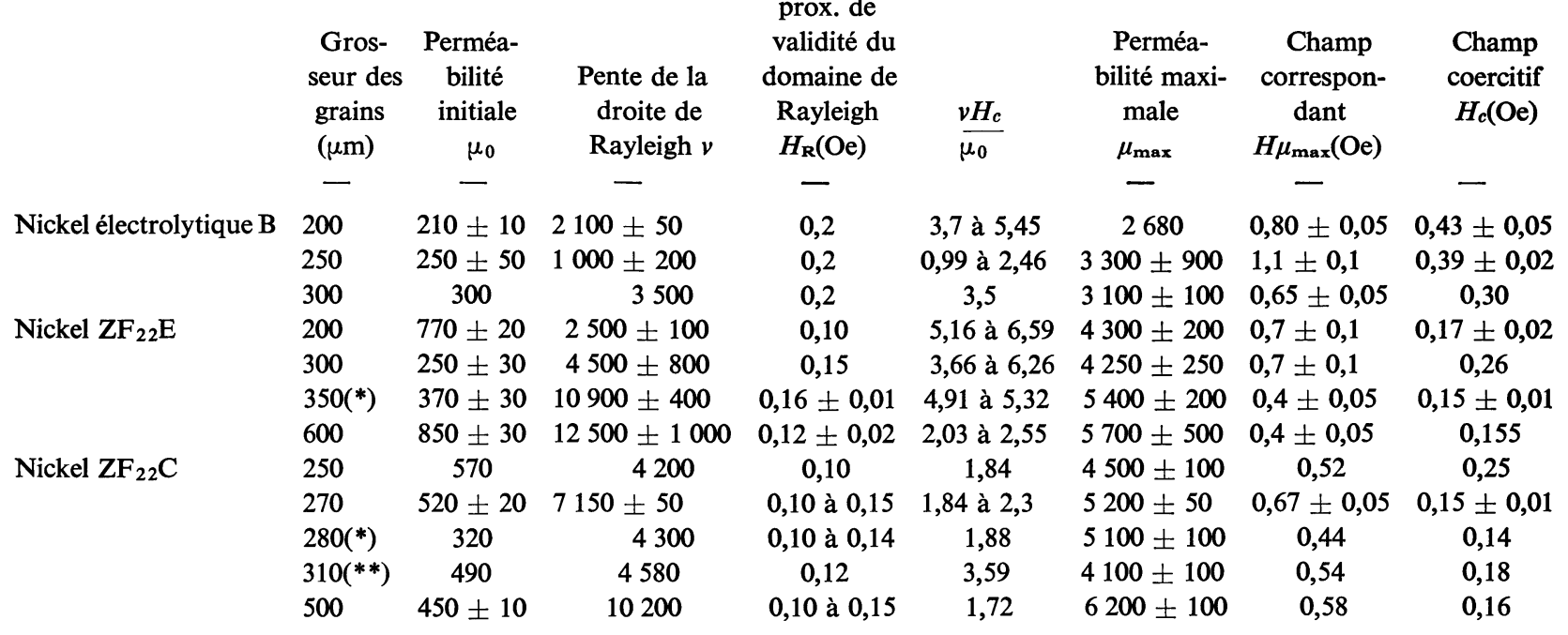

${ }^{*}$ ) Echantillons recuits $8 \mathrm{~h}$ sous hydrogène à $1100^{\circ} \mathrm{C}$.

(**) Echantillon prélevé au milieu du barreau de zone fondue $(x=16 \mathrm{~cm})$. Tous les autres sont prélevés dans les 7 premiers centimètres.

n'avons pas trouvé de signification précise du champ $H \mu_{\max }$ : il reste toujours sensiblement supérieur au double du champ coercitif dans le cas des fils tandis que pour les couronnes on a seulement $H \mu_{\max }>H_{\mathrm{c}}$. Par exemple pour une couronne de nickel $\mathrm{ZF}_{22} \mathrm{C}$ recuite à $950^{\circ} \mathrm{C}$ sous argon pendant $12 \mathrm{~h}$ nous avons trouvé $H \mu_{\max }=0,35$ Oe pour $\mu_{\max }=9000$ mais avec $H_{\mathrm{c}} \simeq 0,2$ Oe [1]. Notons enfin que dans le cas des fils étudiés, la valeur de $\mu_{\max }$ croît à peu près régulièrement avec la taille de grain tandis que $H \mu_{\max }$ et $H_{c}$ présentent une décroissance moins continue.

Les valeurs de l'induction à saturation et de l'induction à la rémanence de fils ne sont pas très aisées à déterminer : d'une part il faut disposer de champs élevés et d'autre part le cycle présente souvent son axe vertical incliné sur l'axe des champs et des corrections sont nécessaires. Aussi les valeurs que nous donnons dans le tableau III sont-elles essentiellement comparatives.

\section{TABLEAU III}

$$
B_{\mathrm{s}}
$$

Nickel

$$
\mathrm{H}=175 \mathrm{Oe} \quad B_{\mathrm{r}}
$$

électrolytique $B \quad 5600$

Nickel $\mathrm{ZF}_{22} \mathrm{C} \quad 6150-6280$

$$
2320-2350
$$$$
4260
$$

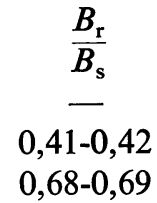

Nous remarquons une valeur plus élevée du rapport $B_{\mathrm{r}} / B_{\mathrm{s}}$ dans le cas du métal de zone fondue. De même pour une couronne de nickel $\mathrm{ZF}_{22} \mathrm{C}$ le rapport $B_{\mathrm{r}} / B_{\mathrm{s}}$ approche $0,8\left(B_{\mathrm{s}}=6750\right.$ pour $\left.H=16,6 \mathrm{Oe}\right)$. Ces résultats suggèrent une structure magnétique de rémanence similaire à la structure proche de la satu- ration, c'est-à-dire une germination plus difficile des domaines d'aimantation inverse dans le métal très pur.

Une application de l'étude précédente réside dans l'utilisation d'une caractéristique magnétique (le champ coercitif $H_{\mathrm{c}}$ par exemple) comme critère de pureté du nickel. La figure 6 reproduit les variations du rapport de résistivités $\rho_{H}$ et de $H_{\mathrm{c}}$ pour des fils de nickel carbonyle prélevés le long d'un barreau ayant subi 6 passages de fusion de zone. La variation de $\boldsymbol{H}_{\mathrm{c}}$ se modèle bien sur celle de $\rho_{H}$, critère habituel de la pureté globale.

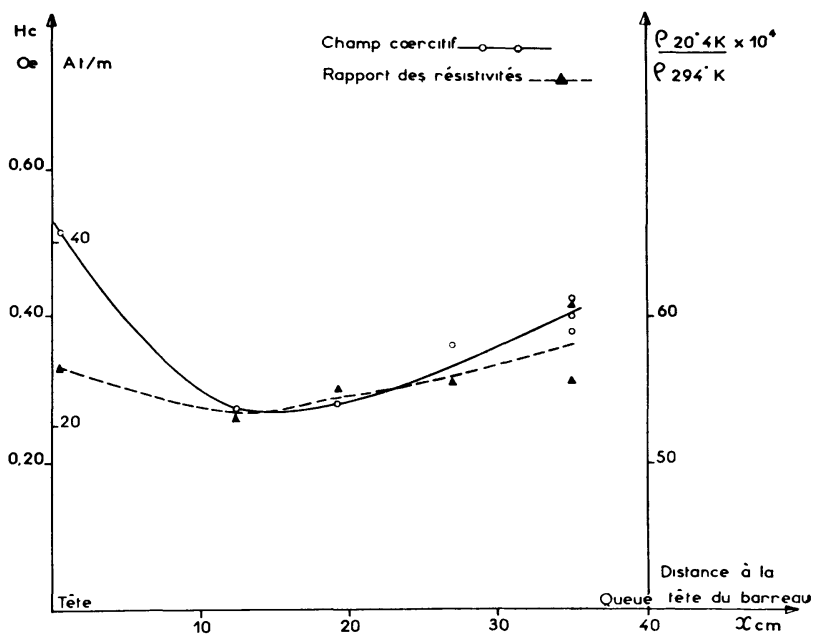

FIG. 6. - Evolutions comparées du champ coercitif $H_{\mathrm{c}}$ et du rapport $\rho_{H}$ pour le nickel d'origine carbonyle traité par 6 passages de fusion de zone.

2.2.3 Influence spécifique de quelques impuretés. L'adoucissement magnétique des métaux de zone 
fondue se manifeste aussi bien dans la subdivision en domaines que dans les valeurs des caractéristiques magnétiques. Mais le métal électrolytique $B$, globalement plus pur que le métal de fusion de zone $\mathrm{ZF}_{22} \mathrm{E}$, présente des caractéristiques magnétiques inférieures (les températures de recristallisation identiques sont explicables par les valeurs voisines des rayons atomiques [1]). Le fait que le soufre soit un poison bien connu du nickel nous a incité à rechercher si cet élément ne serait pas à l'origine des différences observées.

Rôle du soufre. - Le métal électrolytique B possède une teneur en soufre supérieure à celle des métaux de fusion de zone, mais du fait de sa préparation industrielle il est hétérogène. Nous avons effectué la fusion au four à induction de ce métal, en nacelle de chaux, soit sous argon commercial ayant traversé un piège à oxygène liquide, soit sous hydrogène pur et sec. Les lingots obtenus après fusion de $30 \mathrm{mn}$ et solidification rapide sont aisément tréfilés. Les études ont été effectuées au moyen des mesures magnétiques et de la détermination du rapport des résistivités $\rho_{H}$. Les résultats obtenus sont portés dans le tableau IV (en regard de la teneur en soufre) et comparés, à même grosseur de grain, à ceux obtenus sur le métal électrolytique $\mathrm{B}$ et sur le métal de zone fondue $\mathrm{ZF}_{22} \mathrm{E}$.

Tout d'abord la fusion du nickel B sous argon se traduit par une pollution et simultanément une dégradation des propriétés magnétiques. Il est possible que l'argon, insuffisamment purifié, en soit l'origine, mais nous n'avons pas fait d'autres essais. Par contre, il semble bien, dans l'autre cas, qu'une diminution de la teneur en soufre se traduise par une amélioration des propriétés magnétiques (malgré une légère augmentation de $\rho_{H}$ ). Pour préciser ce point, nous avons effectué les opérations décrites sur la figure 7. Dans une première série d'essais, la tranche de lingot de nickel $\mathrm{B}$ fondu sous hydrogène est divisée comme l'indique le schéma (1). Les déterminations de $H_{c}$ et de $\mu_{\max }$ conduisent à des valeurs reproductibles mais différentes pour les moitiés $\mathrm{A}$ et $\mathrm{B}$. La taille de grain importante laisse penser à l'influence possible d'une
(1) _Tréfılage dırect

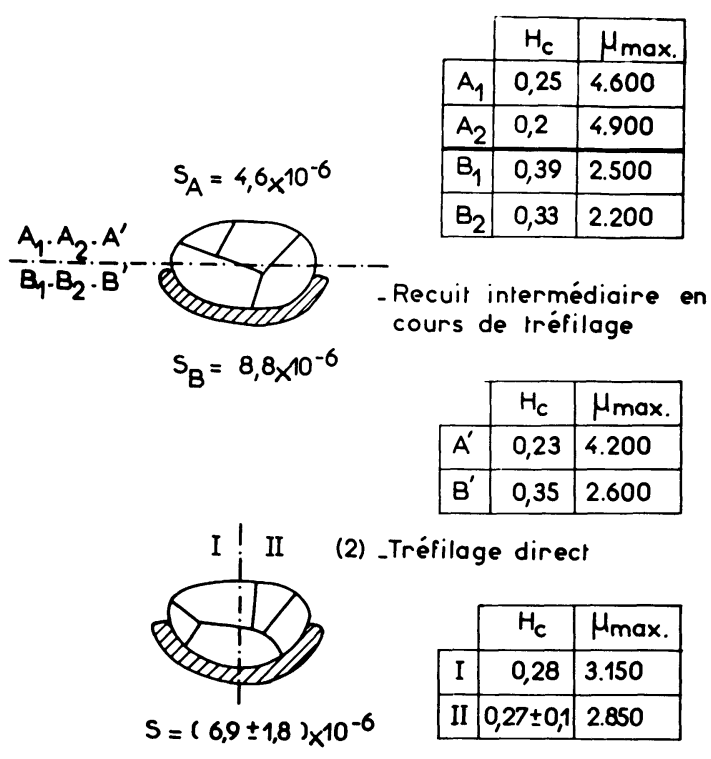

FIG. 7. - Etude analytique du nickel électronique B fondu sous hydrogène.

texture : nous avons alors modifié le taux d'écrouissage en introduisant un recuit intermédiaire à $1200^{\circ} \mathrm{C}$ en cours de tréfilage. Après le recuit final, on retrouve sensiblement les mêmes valeurs pour les deux parties du lingot. Dans une deuxième série d'essais, la tranche de lingot a été découpée suivant le schéma (2). Dans ce cas, les caractéristiques sont les mêmes pour les deux fils.

Nous avons trouvé une explication des valeurs divergentes des propriétés magnétiques dans le dosage du soufre [12]. Le dosage a été effectué dans les parties $A$ et $B$ prises séparément. Nous trouvons pour la moyenne de 3 dosages, respectivement $4,6 \times 10^{-6}$ et $8,8 \times 10^{-6}$ de soufre (ce qui peut indiquer une pollution par la nacelle). Le dosage du soufre dans une section du lingot conduit à $(6,9 \pm 1,8) \times 10^{-6}$ de soufre (pour 4 essais), valeur qui est sensiblement la moyenne des teneurs obtenues pour les fractions A et B. Nous remarquons alors que les valeurs du champ coercitif

TABLEAU IV

\begin{tabular}{|c|c|c|c|c|c|c|}
\hline & $\begin{array}{l}\text { Grosseur } \\
\text { de grain } \\
\left(\mu_{\mathrm{m}}\right)\end{array}$ & $\begin{array}{c}H_{\mathrm{c}}(\mathrm{Oe}) \\
-\end{array}$ & $\begin{array}{r}\mu_{\max } \\
-\end{array}$ & $\rho_{H}=10^{-4} \frac{\rho 20,3 \mathrm{~K}}{\rho 294 \mathrm{~K}}$ & $\begin{array}{c}\text { méthode } \\
\text { d'évolution } \\
\text { [12] }\end{array}$ & $\begin{array}{l}\text { spectro- } \\
\text { graphie } \\
\text { de masse } \\
\end{array}$ \\
\hline $\begin{array}{l}\text { Nickel électro- } \\
\text { lytique B }\end{array}$ & 180 & 0,35 & $2370 \pm 70$ & 130 & 20 & 17 \\
\hline $\begin{array}{l}\text { Nickel B fondu } \\
\text { sous argon } \\
\text { Nickel B fondu }\end{array}$ & $150-200$ & 0,43 & $1750 \pm 50$ & 160 & 50 & - \\
\hline sous hydrogène & $150-200$ & 0,28 & $3000 \pm 150$ & 136 & 5 à 9 & - \\
\hline Nickel $\mathrm{ZF}_{22} \mathrm{E}$ & 200 & $0,17 \pm 0,02$ & $4300 \pm 200$ & 370 & $3 \pm 1,5$ & $\begin{array}{l}4,1 \\
8,6\end{array}$ \\
\hline
\end{tabular}


$H_{\mathrm{c}}$ et de la perméabilité maximale suivent sensiblement cette loi de la moyenne. Nous mettons ainsi en évidence le lien intime qui relie les propriétés magnétiques du nickel à sa teneur en soufre sans toutefois préciser sous quelle forme il se trouve dans le métal. Durant tous les essais que nous venons de décrire, nous avons toujours obtenu une valeur constante à $5 \%$ près du rapport des résistivités $\rho_{H}$.

Influence du cobalt. - Le métal $\mathrm{ZF}_{22} \mathrm{E}$ qui contient $0,7 \%$ de cobalt et $0,1 \%$ de fer présente d'excellentes propriétés magnétiques dues en particulier à sa faible teneur en soufre. Cependant l'influence du cobalt et $\mathrm{du}$ fer se manifeste vis-à-vis de la température de Curie. Pour le nickel $\mathrm{ZF}_{22} \mathrm{E}$, cette température se situe à $367^{\circ}$ au lieu de $357^{\circ} \pm 1^{\circ} \mathrm{C}$. Ce résultat est conforme aux données bibliographiques [18].

La pureté n'est pas le seul facteur qui conditionne les propriétés d'un métal ferromagnétique, les traitements mécaniques et thermiques jouent un rôle important sans oublier les irradiations par particules étudiées au C. E. N. G. [19]. Cependant il est difficile parfois de dissocier le rôle de ces traitements de celui des impuretés.

3. Influence des traitements mécaniques et thermiques. - Par traitements mécaniques nous entendons la mise en forme de l'échantillon avant recuit, mise en forme qui détermine à la fois ses dimensions, son taux d'écrouissage et sa texture de déformation. Nous nous sommes attachés à suivre l'évolution des propriétés magnétiques de fils écrouis en fonction du temps et de la température de traitement thermique.

3.1 EVOLUTION DES PROPRIÉTÉS MAGNÉTIQUES DU NICKEL ÉCROUI EN FONCTION DE LA TEMPÉRATURE DE RECUIT. - Les fils de nickel électrolytique $B$ et de nickel de zone fondue $\mathrm{ZF}_{22} \mathrm{C}$ utilisés pour cette étude, de diamètre $1,2 \mathrm{~mm}$, ont été tout d'abord recuits à $1200{ }^{\circ} \mathrm{C}$ pendant $12 \mathrm{~h}$ sous argon purifié. Puis ils ont été tréfilés de sorte que le taux de réduction de section $\left(S_{0}-S / S_{0}\right)\left(S_{0}\right.$ section initiale, $S$ section

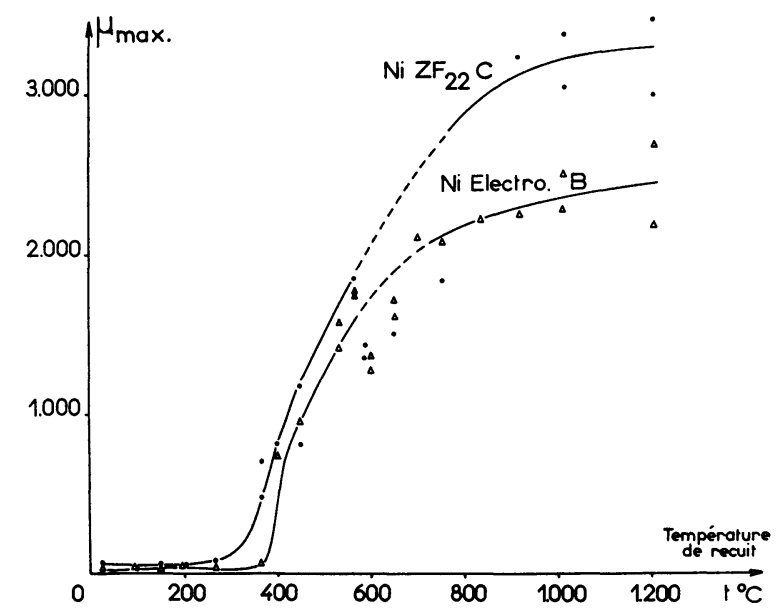

FIG. 8. - Variation de la perméabilité maximale $\mu_{\max }$ avec la température de recuit. finale) soit $80 \%$. Les fils obtenus, de diamètre $0,55 \mathrm{~mm}$ ont une longueur de $200 \mathrm{~mm}$; ils sont portés directement à la température de recuit choisie sous atmosphère d'argon, pendant $12 \mathrm{~h}$. Après refroidissement lent au four $\left(3^{\circ} \mathrm{C} / \mathrm{mn}\right)$, nous déterminons $\mu_{\max }$ et $\boldsymbol{H}_{\mathrm{c}}$. Les résultats sont portés sur les figures $8,9,10$. Nous

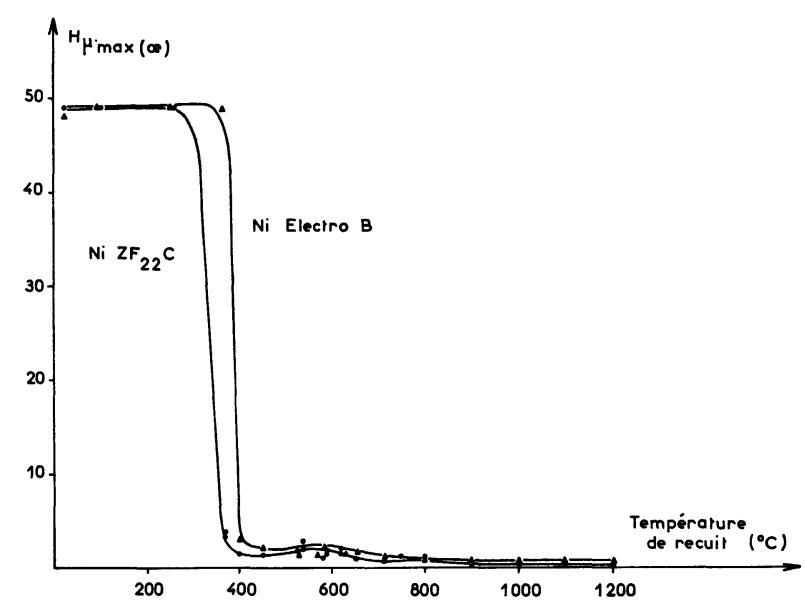

FIG. 9. - Variation du champ $H \mu_{\max }$ avec la température de recuit.

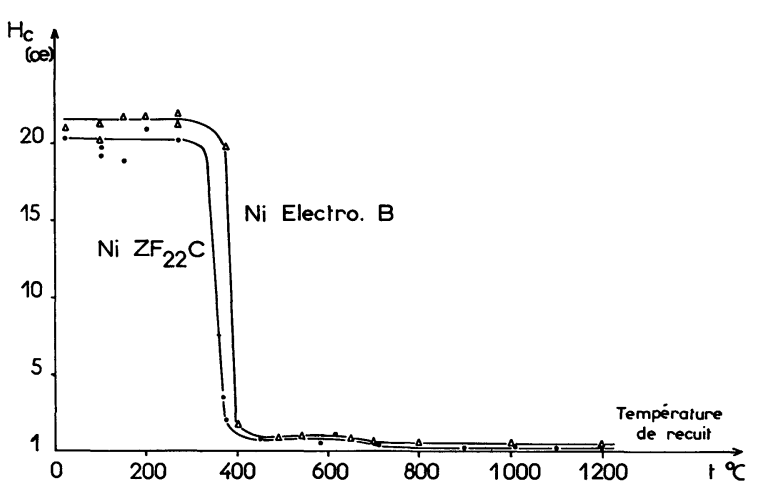

FIG. 10. - Variation du champ coercitif $H_{\mathrm{c}}$ avec la température de recuit.

observons en premier lieu que $\mu_{\max }, H \mu_{\max }$ et $H_{\mathrm{c}}$ ne varient pas jusqu'à la recristallisation, phénomène auquel $H_{\mathrm{c}}$ et $H \mu_{\max }$ sont particulièrement sensibles. Des mesures d'amortissement interne et de résistivité effectuées sur les mêmes fils [1,20] montrent la présence de stades de restauration précédant la recristallisation. De tels stades n'apparaissent pas dans la variation avec la température de recuit des grandeurs étudiées. Il en est de même si l'on étudie la variation des caractéristiques du domaine de Rayleigh sur une couronne de nickel $\mathrm{ZF}_{22} \mathrm{C}$ découpée dans une tôle écrouie de $50 \%$ (Fig. 11). Des résultats semblables ont été trouvés dans le cas du champ coercitif [21] et il semble donc bien que les grandeurs $H_{\mathrm{c}}, H \mu_{\max }$, $\mu_{\max }, \mu_{0} \ldots$ ne soient essentiellement sensibles qu'à une élimination massive des dislocations. Ceci est à rapprocher des conclusions de Kronmüller et Seeger [22] à la suite d'études relatives au champ coercitif 


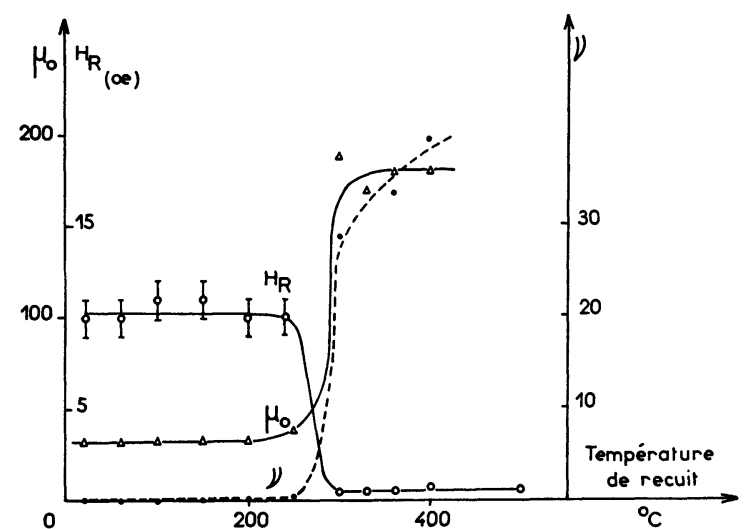

Fig. 11. - Variations des caractéristiques principales du domaine de Rayleigh avec la température de recuit (couronne de nickel $\mathrm{ZF}_{22} \mathrm{C}$ ).

du nickel faiblement déformé. Dans l'intervalle de températures de recuit compris entre $500^{\circ} \mathrm{C}$ et $800^{\circ} \mathrm{C}$, nous notons une dégradation importante des propriétés étudiées, en particulier de la perméabilité maximale. Nous avons attribué les perturbations observées à une intervention des impuretés au cours des traitements thermiques. Les impuretés mises en cause peuvent être contenues dans le nickel ou provenir de l'atmosphère de recuit ou bien encore avoir été introduites lors de la mise en forme des échantillons. Ces impuretés pourront soit participer à des réactions chimiques (réaction avec l'atmosphère), soit à des phénomènes de diffusion ou d'adsorption, soit enfin modifier leur répartition dans le réseau (mise en solution ou précipitation). Les perturbations observées dans ce domaine de température ne sont pas propres aux propriétés magnétiques mais se retrouvent dans le cas de la résistivité, du frottement interne [20] et des propriétés mécaniques [23]. En particulier, les mesures de résistivité électrique à basse température indiquent que la technique de mise en forme des échantillons ne peut être une cause majeure du phénomène mais que par contre, il est possible d'incriminer l'atmosphère d'argon utilisée pour le recuit [20]. Enfin, au-dessus de $800^{\circ} \mathrm{C}$, on retrouve une variation régulière des propriétés magnétiques avec la température de recuit, le métal de fusion de zone ayant la valeur la plus élevée pour $\mu_{\max }$ et la plus basse pour $H_{\mathrm{c}}$ (Fig. 13).

3.2 INFLUENCE DU TEMPS DE TRAITEMENT THERMIQUE. - Nous avons étudié la variation du champ coercitif $H_{\mathrm{c}}$ pour deux nuances de nickel en fonction du temps de traitement à $1200^{\circ} \mathrm{C}$. Par durée du traitement thermique, nous entendons le fil écroui de $80 \%$ introduit directement dans le four à $1200{ }^{\circ} \mathrm{C}$ sous argon purifié, maintenu pendant une heure, puis refroidi lentement. Les résultats présentés sur la figure 12 sont relatifs au nickel électrolytique $\mathrm{B}$ et au nickel de fusion de zone $Z F_{22} C$. Nous observons que le champ coercitif de ce dernier métal prend plus rapidement une valeur constante. Au bout de $6 \mathrm{~h}$

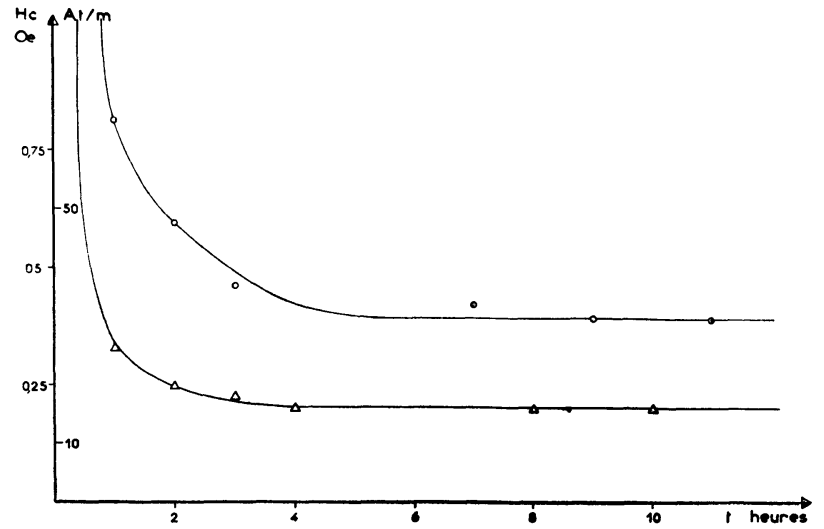

FIG. 12. - Evolution du champ coercitif avec le temps de maintient à $1200^{\circ} \mathrm{C}$

- - - - Nickel électrolytique $B$.

$-\Delta-\Delta$ - Nickel $Z_{2}{ }_{22} C$.

de recuit on peut considérer que $H_{\mathrm{c}}$ est constant pour les deux nuances : ainsi nos caractéristiques magnétiques données pour un recuit de $12 \mathrm{~h}$ correspondent à un état bien recuit du nickel. Si l'atmosphère de recuit est parfaitement neutre, le champ coercitif permet de suivre la cinétique d'élimination des défauts réticulaires, principalement des dislocations. La restauration du champ coercitif d'un nickel écroui de $19 \%$ a été étudiée par Hayashi et Fujimura [24] qui ont trouvé une proportionnalité entre $H_{\mathrm{c}}$ et la racine carrée de la densité de dislocations. Cependant des phénomènes chimiques (évaporation, réactions gaz-métal...) interviennent ainsi qu'une évolution de la taille du grain du métal recristallisé.

3.3 Effet de la taille de Grain. - Nous avons indiqué au paragraphe 2 que l'augmentation de la grosseur de grain, du fait de la plus grande mobilité des parois, se traduisait par de meilleures caractéristiques magnétiques (dans le sens d'un adoucissement). La taille de grains est en relation intime avec la pureté du métal, mais elle dépend aussi des facteurs mécaniques, géométriques, et thermiques. Ainsi nous avons constaté (Fig. 13) dans le cas de fils de $0,55 \mathrm{~mm}$ de diamètre de nickel $\mathrm{ZF}_{22} \mathrm{C}$, que $H_{\mathrm{c}}$ et $\mu_{\max }$ ne varient

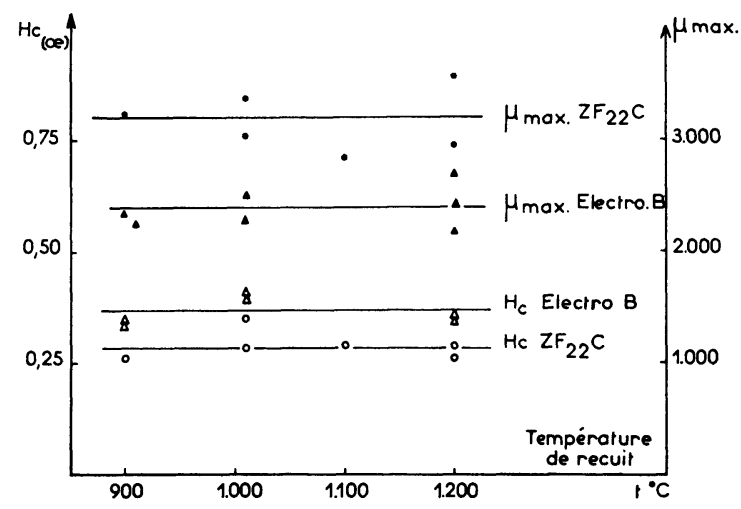

FIG. 13. - Variation de la perméabilité $\mu_{\max }$ et du champ coercitif $H_{c}$ de fils fins recuits entre $900^{\circ} \mathrm{C}$ et $1200^{\circ} \mathrm{C}$. 
pas sensiblement, bien que la taille de grain passe de $200 \mu \mathrm{m}$ (recuit à $900^{\circ} \mathrm{C}$ ) à $350 \mu \mathrm{m}$ (recuit à $1200^{\circ} \mathrm{C}$ ).

Des déterminations de la magnétostriction à saturation $\lambda_{\mathrm{s}}$ ont été effectuées au Laboratoire de Magnétisme de Bellevue [5]. Pour le nickel écroui, quelle que soit sa pureté on trouve $\lambda_{\mathrm{s}}=-33 \times 10^{-6}$ (si la charge reste inférieure à $100 \mathrm{~g} / \mathrm{mm}^{2}$ ). Si le nickel est recuit, on trouve $\lambda_{\mathrm{s}}=-(36 \pm 1) \times 10^{-6}$ pour le nickel $A$ très impur (tableau $I)$ et $\lambda_{s}=-(37 \pm 1) \times 10^{-6}$ pour le nickel de zone fondue $\mathrm{ZF}_{22} \mathrm{C}$. La magnétostriction serait indépendante de la pureté mais dépendrait de l'état structural. Cependant, dans le cas du métal de zone fondue les valeurs de $\lambda_{\mathrm{s}}$ mesurées lorsque le champ est parallèle à l'axe et lorsqu'il est perpendiculaire ne sont pas dans le rapport $-\frac{1}{2}$ prévu par la théorie et retrouvé dans le cas du métal A. Ceci pourrait être en relation avec la présence de grains plus gros dans le métal pur introduisant une distribution anisotrope de l'aimantation.

Nous avons déjà noté que la texture pouvait jouer un rôle important mais nous n'avons pas précisé comment les textures du nickel [25] influencent les propriétés magnétiques.

3.4 INFLUENCE DE L'ATMOSPHÈRE DE RECUIT. Des essais de recuit du nickel ont été effectués sous vide sous argon et sous hydrogène. Par recuit, sous vide de $10^{-6}$ torr, en tube de silice, nous avons observé une pollution se traduisant par une augmentation anormale de la résistivité. L'atmosphère d'hydrogène convient parfaitement pour le recuit des métaux de fusion de zone, mais les métaux industriels présentent un gonflement incompatible avec l'étude des propriétés magnétiques. Les recuits sous argon purifié nous ont paru convenir pour des études comparées entre métaux purs et métaux industriels. Si l'argon n'est pas purifié, il apparaît sur la surface du métal une striation superficielle qui empêche le dépôt de la magnétite utilisée pour révéler les parois de Bloch. Cependant, dans le cas de traitement sous argon purifié, la température de recuit joue un rôle important. Nous avons déjà signalé qu'entre $600{ }^{\circ} \mathrm{C}$ et $900^{\circ} \mathrm{C}$, des variations aléatoires des propriétés magnétiques se produisaient en relation avec un maximum de résistivité caractéristique d'une pollution. En utilisant le métal de fusion de zone $\mathrm{ZF}_{8} \mathrm{~L}$, de très haute pureté (tableau I), nous avons trouvé les mêmes valeurs des propriétés magnétiques pour des fils de $0,55 \mathrm{~mm}$ de diamètre recuits à $1000^{\circ} \mathrm{C}$ sous hydro- gène et à $1200^{\circ} \mathrm{C}$ sous argon purifié. Par contre, des fils recuits à $800{ }^{\circ} \mathrm{C}$ sous argon ou sous hydrogène purifiés voient leurs propriétés magnétiques se dégrader comme l'indique le tableau V (la grosseur de grain est $500 \mu \mathrm{m}$ ).

Nous observons une modification plus faible des propriétés magnétiques après traitement à $800{ }^{\circ} \mathrm{C}$ sous hydrogène, sans que la résistivité en soit très affectée. Dans le cas d'un fil de nickel $\mathrm{ZF}_{8} \mathrm{~L}$, de diamètre $1,2 \mathrm{~mm}$, recuit une heure sous argon purifié à $1200{ }^{\circ} \mathrm{C}$, nous trouvons $H_{\mathrm{c}}=0,24 \mathrm{Oe}$; si nous reportons le même fil une heure à $700^{\circ} \mathrm{C}$ sous argon purifié nous trouvons $H_{\mathrm{c}}=0,27 \mathrm{Oe}$. Ce résultat nous incite à penser à une pollution d'autant moins intense que le fil est plus épais. L'origine de cette pollution pourrait être trouvée dans la présence de traces d'oxygène dans l'argon purifié : en effet la solubilité de l'oxygène dans le nickel diminue de $200 \times 10^{-6}$ à $120 \times 10^{-6}$ lorsque la température s'élève de $600^{\circ} \mathrm{C}$ à $1200^{\circ} \mathrm{C}$ [26]. Mais d'autres métalloïdes peuvent être mis en cause, en particulier le soufre, le carbone qui, avec l'oxygène, seraient à l'origine de la fragilité du nickel recuit entre $600^{\circ} \mathrm{C}$ et $800^{\circ} \mathrm{C}$ [23] [27] [28]. Une étude remarquable a été effectuée récemment sur les ségrégations intergranulaires dans le nickel industriel, mais le problème reste complexe [29].

3.5 IMPORTANCE DES DIMENSIONS GÉOMÉTRIQUES DES ÉCHANTILLONS. - Il est indispensable, lors de la fabrication des fils soumis à des essais magnétiques, de soigner particulièrement leur surface pour éviter les champs de dispersion. D'autre part, les dimensions du fil imposent un certain nombre de corrections de mesures [1]. Nous voudrions apporter quelques résultats relatifs à cet effet de dimensions. Nous avons comparé après le même traitement thermique, les caractéristiques magnétiques de fils longs de $20 \mathrm{~cm}$, de diamètres $1,2 \mathrm{~mm}$ et $0,55 \mathrm{~mm}$. Les résultats qui figurent dans le tableau VI ont pu être reproduits sur plusieurs dizaines de fils.

La diminution de diamètre semble affecter le champ coercitif $H_{\mathrm{c}}$ de façon un peu plus importante que la perméabilité $\mu_{\max }$, l'effet étant le plus sensible sur le métal le plus pur. Remarquons que, d'après le paragraphe précédent, une dégradation plus marquée des caractéristiques magnétiques apparaît sur les fils les plus fins. Nous avons étudié systématiquement la variation du champ coercitif $H_{\mathrm{c}}$ pour des fils de diamètres différents de nickel $\mathrm{B}$ et de nickel $\mathrm{ZF}_{22} \mathrm{C}$.

TABLEAU V

Nickel $\mathrm{ZF}_{8} \mathrm{~L}$
recuit $90 \mathrm{mn}$
$\mu_{\max }$
$H \mu_{\max }(\mathrm{Oe})$
$H_{\mathrm{c}}(\mathrm{Oe})$
$10^{4} \times \rho_{H}$

$1200{ }^{\circ} \mathrm{C}$
sous argon
4130
0,42
0,294
26

\section{$800^{\circ} \mathrm{C}$} sous argon

785

4,3

2,76

32,5
$1000{ }^{\circ} \mathrm{C}$ sous hydrogène

4750

0,44

0,34
$800{ }^{\circ} \mathrm{C}$ sous hydrogène

1920

1,12

0,71 
TABleau VI

Nickel électrolytique B diamètre

$$
300
$$

Grosseur de grain (en $\mu \mathrm{m}) \ldots$

Champ coercitif $H_{\mathrm{c}}(\mathrm{Oe}) \ldots \ldots$

Perméabilité maximale $\mu_{\max }$. .

Champ corresp. $H \mu_{\max }(\mathrm{Oe}) \ldots$

Perméabilité initiale $\mu_{\mathrm{o}} \ldots \ldots$.

Pente de la droite de Rayleigh

Validité du domaine de Rayleigh

$$
\begin{gathered}
\text { diamètre } \\
0,55 \mathrm{~mm} \\
- \\
250 \\
0,345 \\
2700 \\
0,70 \\
220 \\
250 \text { à } 650 \\
0,2
\end{gathered}
$$

Nickel zone fondue $\mathrm{ZF}_{22} \mathrm{C}$ diamètre diamètre $1,2 \mathrm{~mm}$ $0,55 \mathrm{~mm}$

$31 \overline{0}$
0,185
4100
0,54
490
4200 à 10200
0,12

600 à 1800 0,12

Les résultats sont portés sur la figure 14. Lorsque le diamètre d'un fil diminue, il se produit un accroissement des champs de dispersion superficiels. Ces champs apparaissent

- soit à l'aplomb de la paroi de Bloch et provoquent l'augmentation de l'énergie de paroi [30] ;

- soit à la surface des domaines dont la direction de facile aimantation n'est pas dans le plan de surface [31].

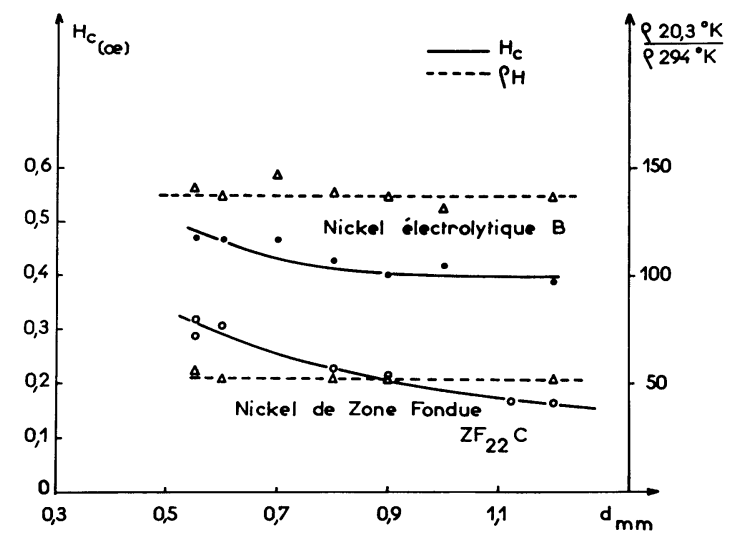

FIG. 14. - Comparaison en fonction du diamètre de fil de $\rho_{H}$ et de $H_{c}$ (taille du grain $270 \mu \mathrm{m}$ pour le métal B et $330 \mu \mathrm{m}$ pour le métal $\mathrm{ZF}_{22} \mathrm{C}$ ).

Remarquons que l'augmentation du champ coercitif $H_{\mathrm{c}}$ quand le diamètre diminue est plus marquée dans le cas du nickel le plus pur, ce qui pourrait s'expliquer par une plus grande taille des domaines (toutefois l'observation des domaines sur des fils de tels diamètres n'est pas très aisée). Ces résultats sont à rapprocher de ceux de Katrus [32] obtenus sur des bandes de nickel moins pur préparé par frittage : l'augmentation de $H_{\mathrm{c}}$ ne se produit que pour des épaisseurs inférieures à $0,5 \mathrm{~mm}$. Remarquons que pour chaque nuance de nickel, la résistivité ne varie pas dans la gamme de diamètres envisagés.

3.6 ESSAIS DE TRAINAge MAGNÉTIQUe. - Nous avons entrepris quelques essais de traînage magnétique en courant alternatif [6] [7] : on fixe la tension d'entrée $V_{0}$ et l'on étudie la tension de sortie $V_{i}$ en fonction de la température. Les expériences relatives

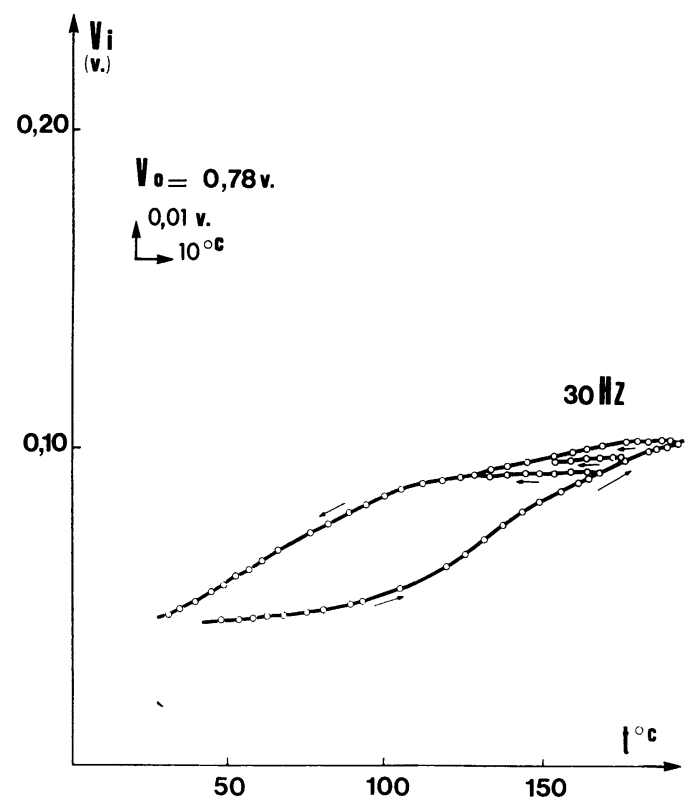

(a)

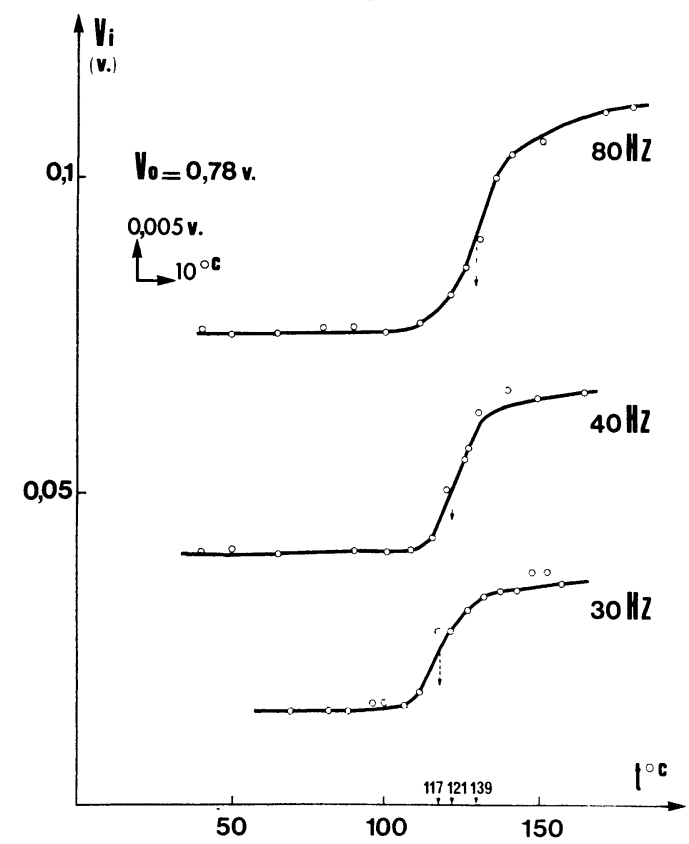

(b)

Fig. 15. - Influence d'une déformation sur la variation avec la température du traînage magnétique du nickel d'origine carbonyle.

a) recuit 1 h. à $1000^{\circ} \mathrm{C}$.

b) allongé de $5 \%$. 
au nickel ont été effectuées sur des fils (diamètre $1 \mathrm{~mm}$, longueur $200 \mathrm{~mm}$ ) de nickel d'origine carbonyle, à teneur relativement importante en métalloïdes $\left(\mathrm{S}: 27 \times 10^{-6}-\mathrm{C}: 50 \times 10^{-6}\right)$. Les fils ont été recuits $1 \mathrm{~h}$ à $1000^{\circ} \mathrm{C}$ sous vide de $10^{-5}$ torr.

Entre l'ambiante et $180^{\circ} \mathrm{C}$, pour une fréquence de $30 \mathrm{~Hz}$, nous avons tracé les variations de $V_{i}$ avec la température au chauffage et au refroidissement (Fig. 15a). On décrit ainsi un cycle d'hystérésis pratiquement complet mais l'aspect des courbes est semblable aux variations de la perméabilité initiale avec la température [33,34].

De nombreux travaux ont déjà été consacrés au traînage magnétique, et en particulier à son utilisation pour l'étude des défauts ponctuels [38]. Nous avons envisagé d'introduire des dislocations en étirant les fils recuits de $5 \%$. Les courbes obtenues sont portées sur la figure $15 b$. Nous remarquons tout d'abord une diminution de $V_{i}$ correspondant à une diminution de la perméabilité initiale avec le degré d'écrouissage. De plus les courbes obtenues présentent une inflexion nettement plus marquée qu'avant écrouissage et ressemblent ainsi à celles obtenues lors de l'étude du carbone dans le fer [6,7]. Ce dernier effet, d'autant plus marqué que le nickel est moins pur, peut s'exprimer en termes d'interactions entre parois de Bloch et impuretés métalloïdiques (l'énergie d'activation déduite des courbes de la figure $15 b$ est de l'ordre de $1 \mathrm{eV})$.

4. Intervention des propriétés magnétiques dans les mesures de résistivité électrique à basse température et dans la détermination de la capacité d'amortissement interne du nickel. - Les mesures de résistivité électrique à basse température permettent une évaluation globale de la pureté d'un métal bien recuit, car l'on minimise à la fois la résistivité due à la diffusion des électrons par les phonons et de celle due aux défauts physiques. Cependant dans le cas d'un ferromagnétique, il s'ajoute un excès de résistivité de nature magnétique et difficile à préciser. Pour s'affranchir de ce terme, on étudie la résistivité en fonction de l'intensité d'un champ continu longitudinal et on choisit comme critère de pureté la valeur extrapolée à champ nul. Si le nickel est impur, la différence entre valeur mesurée en l'absence de champ et valeur extrapolée est faible. Dans le cas du nickel $\mathrm{ZF}_{8} \mathrm{~L}$ (tableau I). Ces valeurs diffèrent d'environ $10 \%$ alors que pour le fer de haute pureté la différence peut atteindre $50 \%$ [39]. Nous pouvons donc dire que le rapport des résistivités électriques $\rho 4,2 \mathrm{~K} / \rho 294 \mathrm{~K}$ est un critère valable de la pureté globale du nickel.

Lors de mesures d'amortissement interne effectuées au pendule de Ké (mesure du décrément logarithmique des oscillations libres du pendule), nous avons constaté un effet important lors de l'application d'un champ longitudinal. Si nous traçons la variation de l'amortissement interne avec l'amplitude de déformation, nous observons une diminution de l'amor- tissement interne avec l'intensité du champ, diminution d'autant plus importante que le métal est plus pur : les domaines participent à l'amortissement des oscillations mécaniques (Fig. 16). La variation de l'amortissement interne à champ nul est linéaire en fonction de l'amplitude quand le nickel est impur, ce qui avait incité Kornetzky [40] à faire une analogie avec la loi de Rayleigh. Quand la pureté augmente, le frottement interne croît mais, de plus, la courbe s'incurve légèrement. Sous champ magnétique continu de 300 œrsteds, l'amortissement interne est d'abord indépendant de l'amplitude (A) puis croît linéairement (B) et, enfin, adopte une forme parabolique (C, D) avec peut-être une asymptote dans le cas du métal le plus pur. En comparant les courbes amortissement-amplitude sous champ de $\mathbf{3 0 0}$ œrsteds aux valeurs des résistivités de fils de différentes nuances de nickel, nous avons montré qu'une telle courbe est un critère de la pureté globale du nickel [1].
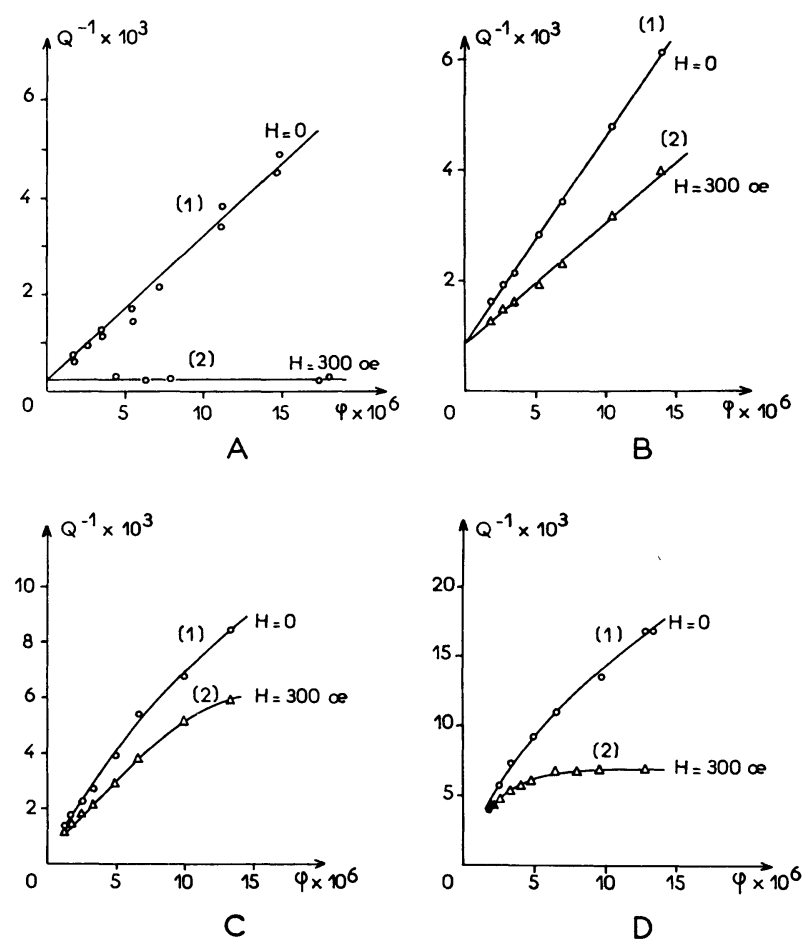

FIG. 16. - Courbes « amortissement interne - amplitude de déformation $»$ de différentes nuances de nickel.

A nickel très impur $\left(\rho_{H}=1570 \times 10^{-4}\right)$

$\mathrm{B}$ nickel $\mathrm{ZF}_{22} \mathrm{E}$

C nickel $\mathrm{B}$

D nickel $\mathrm{ZF}_{22} \mathrm{C}$

Remarquer le changement d'échelle.

Dans le domaine des champs faibles, nous avons précisé la variation de l'amortissement interne avec l'intensité du champ magnétique continu [41]. Un maximum apparaît à toutes les amplitudes pour un champ $H_{Q \max }^{-1}$ difficile à préciser (Fig. 17). Dès 200 œrsteds, l'amortissement interne ne varie plus avec l'intensité du champ. Nous observons que la hauteur du pic, au-dessus de l'amortissement interne à saturation, croît avec l'amplitude. Ce pic apparaît 


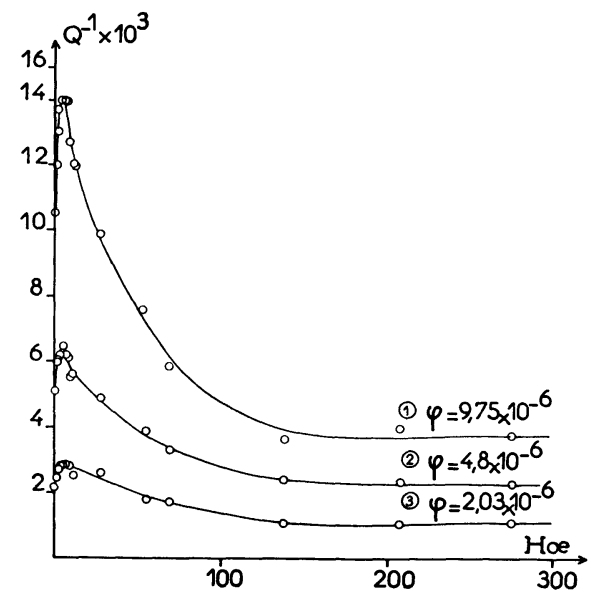

FIG. 17. - Variation du frottement interne du nickel $\mathrm{ZF}_{22} \mathrm{C}$ avec l'intensité du champ magnétique appliqué pour différentes amplitudes de déformation.

pour un champ $H_{Q \max }^{-1}$ de 5 œrsteds dans le métal $\mathrm{ZF}_{22} \mathrm{C}$ et de 10 œrsteds dans le métal électrolytique $\mathrm{B}$, à même amplitude. Pour le nickel $\mathrm{ZF}_{22} \mathrm{C}$, ce champ passe de 5 à 10 œrsteds quand la tension du fil passe de $0,14 \mathrm{~kg} / \mathrm{mm}^{2}$ à $0,27 \mathrm{~kg} / \mathrm{mm}^{2}$, ce qui est analogue aux résultats de Van Neste [42]. Il a été proposé d'identifier ce champ avec $H \mu_{\max }$ [43] : nous pensons plutôt comme Mišek [44] qu'il est seulement possible de calquer ses variations sur celles du champ coercitif $H_{\mathrm{C}}$. Nous avons attribué les phénomènes observés aux interactions compétitives de la tension du fil et du champ appliqué vis-à-vis de l'aimantation [41].

Conclusions. - Les propriétés magnétiques du nickel polycristallin sont conditionnées par de nombreux facteurs. L'observation en microscopie optique de l'aspect des domaines de Weiss reflète bien la pureté globale du nickel : domaines plus étendus et structure plus régulière dans le nickel le plus pur. Mais les mesures indiquent que les propriétés magnétiques du nickel pur sont essentiellement tributaires de la teneur en soufre du métal. Des teneurs de quelque $10^{-6}$ perturbent notablement la perméabilité maximale et le champ coercitif alors que $0,7 \%$ de cobalt n'affectent que la température de Curie. Cependant, même pour du nickel de haute pureté, nous n'avons pas pu trouver entre les paramètres du domaine de Rayleigh une relation analogue à celle obtenue pour le fer et prévue par la théorie.

Les propriétés magnétiques du nickel sont extrêmement sensibles aux traitements thermiques. D'une part la taille grain influence directement la perméabilité maximale. D'autre part, entre 600 et $800^{\circ}$, une atmosphère de recuit très légèrement oxydante provoque une dégradation des propriétés magnétiques analogue à celle observée dans le cas des propriétés mécaniques et électriques. Après recuit à plus haute température, pour une taille de grain donnée, un durcissement magnétique apparaît quand le diamètre du fil diminue et ce durcissement est d'autant plus accentué que le métal est plus pur.

Enfin, après quelques essais de traînage magnétique, nous avons indiqué que les propriétés magnétiques du nickel avaient peu d'influence sur la résistivité électrique à basse température alors qu'elles intervenaient de façon importante dans les mesures d'amortissement interne en fonction de l'amplitude, critères de la pureté globale du nickel.

\section{Bibliographie}

[1] Dubois (B.), Thèse Doctorat Paris, 1966, C. N. R. S. A. O. 1397.

[2] Garrood (J. R.), Proc. Phys. Soc., 1962, 79, 1252.

[3] Dabosi (F.), Mém. Sci. Rev. Mét., 1962, 59, 685 et 784.

[4] Schauer (A.), Zeit. fur Ang. Phys., 1963, 16, 90.

[5] de Lacheisserie (E.), J. Physique, 1966, 27, 555.

[6] Maringer (R. E.), J. App. Phys., 1964, 35, 2375.

[7] Blanc (G.), C. R. Acad. Sci., 1966, 262, 811.

[8] Talbot (J.), Albert (Ph.) et Chaudron (G.), $C . R$. Acad. Sci., 1957, 244, 1577. Dubois (B.), Mém. Sci. Rev. Mét., 1968, 65, 67.

[9] Vu Quang Kinh et Nardin (M.), C. R. Acad. Sci., 1968, 266, 307.

[10] Albert (Ph.), L'analyse par radioactivation, Paris, 1963, Gauthiers Villars.

[11] Durand (J. C.), Chaudron (Th.) et Montuelle (J.), Bull. Soc. Chim., 1965, 11, 3109.

[12] Luke (C. L.), Analytical Chemistry, 1957, 29, 1227.

[13] Dubois (B.), Waché (A. M.), Dabosi (F.) et Talbot (J.), Mém. Sci. Rev. Mét., 1963, 60, 851.

[14] Bloor (D.) et Martin (D. H.), Proc. Phys. Soc., 1959, 73, 694.

[15] Jackson (R. C.), Lee (E. W.) et Troughton (G. H.), Brit. J. App. Phys., 1958, 9, 495.
[16] Kahan (Th.), Ann. Phys. 11 e série, 1938, 9, 105.

[17] NéEl (L.), Cahiers de Physique, 1942, 12, 1, 1943, $13,1$.

[18] Bozorth (R.), Ferromagnetism. Van Nostrand, 1950, $115,280$.

[19] Peretto (P.), Thèse Grenoble, 1967, C. E. N. G. B. P. 269.

Oddou (J. L.), Thèse, Grenoble, 1968, C. E. N. G. B. P. 269.

[20] Dubois (B.) et Dimitrov (O.), Mém. Sci. Rev. Mét., 1967, 64, 641.

[21] Kimanek (P.), Reichel (G.) et Scharf (H.), Phys. Stat. Sol., 1965, 11, 723.

[22] Kronmuller (H.), Seeger (A.), JaGer (H.) et Rieger (H.), Phys. Stat. Sol., 1962, 2, K 105. Seeger (A.) et Kronmuller (H.), Phil. Mag., $1962,7,897$.

[23] Henry (G.), Plateau (J.), Waché (X.), Gerber (Mlle), Behar (I.) et Crussard (C.), Mém. Sci. Rev. Mét., 1959, 56, 417.

[24] Hayashi (S.) et Fujimura (T.), Sci. Rpt. R. I. T. U., A. 16, 1964, 81 .

[25] Clark (C. A.) et Mee (P. B.), Zeit. fur Metallk, 1962, 53, 756. 
[26] Pascal (P.), Nouveau Traité de Chimie Minérale, 1963, 17, 729.

[27] Olsen (K. M.), LaRkin (C. F.) et Schmitt (P. M. Jr), Trans. A. S. M., 1960, 53, 349.

[28] Yamane (T.) et TanaKa (M.), Trans. J. I. M., 1963, 4, 162.

[29] Waché (C.), Thèse Orsay, 1970, Pub. I. R. S. I. D.

[30] Néel (L.), C. R. Acad. Sci, 1955, 241, 533.

[31] Zaikova (V. A.), Shur (Ya. S.) et Falaleyev (G. A.), Phys. of Met. Metall., 1962, 14, 126.

[32] Katrus (O. A.), Phys. of Met. Metall., 1962, 14, 126.

[33] Kersten (M.), Zeit. fur Ang. Phys., 1956, 8, 382.

[34] Rieger (H.), Phys. Stat. Sol., 1965, 8, 283.
[35] NéEl (L.), J. de Physique, 12, 1951, 339. 1952, 13, 249. J. App. Physique, 1959, 30, 15.

[36] Brissonneau (P.), J. de Physique, 1959, 20, 244.

[37] Klein (M. V.) et Kronmuller (H.), J. Appl. phys., 1962, 33, 2191.

[38] Moser (P.), Mém. Sci. Rev. Met., 1966, 63, 1.

[39] Merklen (P.), Mém. Sci. Rev. Met., 1968, 65, 71.

[40] Kornetzky (M.), Z. Phys., 1943, 121, 560.

[41] Dubois (B.), Mém. Sci. Rev. Mét., 1966, 63, 309.

[42] Van Neste (A.), et Dubé (A.), C. R. Acad. Sci., 1965, $260,5019$.

[43] KéKalo (I. B.) et Livshits (B. G.), Phys. Met. Metallo. $1962,13,109$.

[44] MišEK (K.), Czech. J. Phys., 1956, 6, 331. 